\title{
Algebraic methods in control, theory and applications
}

\author{
Vladimír Kučera \\ Institute of Information Theory and Automation \\ Academy of Sciences of the Czech Republic \\ Pod vodárenskou věž $4, P$. O. Box 18, 18208 Praha, CZ. \\ Tel: (42-2) 6884669. Fax: (42-2) 6884903. \\ e-mail: kucera@utia.cas.cz
}

\begin{abstract}
Fractional representations are a useful algebraic tool for control system design. They result in a simple parametrization of all controllers that stabilize a given plant. One could then, in principle, choose the best controller for various applications.
\end{abstract}

\section{Keywords}

Algebraic system theory; control system synthesis; feedback control; linear systems; transfer functions.

\section{INTRODUCTION}

This paper is a tutorial whose aim is to explain a useful algebraic tool for control system design, the so-called fractional representation approach. This approach is based on the input-output properties of linear systems. The central idea is that of representing the transfer function of a (not necessarily stable) system as the ratio of two stable transfer functions. This is a natural step for the linear systems whose transfer functions are rational, i.e., for the lumped-parameter systems. Under certain conditions, however, this approach is productive also for the distributed-parameter systems.

The starting point of the design is to obtain a simple parametrization of all controllers that stabilize a given plant. One could then, in principle, choose the best controller for various applications. The key point is that the parameter appears in the closed-loop system transfer function in a linear manner, thus making it easier to meet additional design specifications.

The actual design of control systems is an engineering task that cannot be reduced to algebra. Design contains many additional aspects which have to be taken into account: sensor placement,computational constraints, actuator constraints, redundancy, performance robustness, among many others. There is a need for understanding of the control process, a feeling for what kinds of performance objectives are unrealistic, or even dangerous, to 
ask for. The algebraic approach to be presented, nevertheless, is an elegant and useful tool for the mathematical part of the controller design (Kučera, 1993).

\section{SYSTEMS AND SIGNALS}

The fundamentals of the fractional representation approach will be explained for linear systems with rational transfer functions whose input $u$ and output $y$ are scalar quantities. We suppose that $u$ and $y$ live in a space of functions mapping a time set into a value set. The time set is the set of non-negative reals, $R_{+}$, and the value set is taken to be the set of real numbers $R$.

Let the input and output spaces of a system be the spaces of locally (Lebesgue) integrable functions $f$ from $R_{+}$into $R$ and define a $p$-norm

$$
\begin{gathered}
\|f\|_{L_{p}}=\left[\int_{0}^{\infty}|f(t)|^{p} d t\right]^{1 / p} \quad \text { if } 1 \leq p<\infty \\
\|f\|_{L_{\infty}}=\text { ess } \sup _{t \geq 0}|f(t)| \quad \text { if } p=\infty .
\end{gathered}
$$

The corresponding normed space is denoted by $L_{p}$.

Systems having the desirable property of preserving the functional space are called stable. More precisely, a system is said to be $L_{p}$-stable if any input $u \in L_{p}$ gives rise to an output $y \in L_{p}$. The systems that are $L_{\infty}$-stable are also termed to be bounded-input bounded-output (BIBO) stable.

The transfer function of a system is the Laplace transform of its impulse response $g(t)$,

$$
G(s)=\int_{0}^{\infty} g(t) e^{-s t} d t .
$$

It is well known that a system with a rational transfer function $G(s)$ is BIBO stable if and only if $G(s)$ is proper and stable, i.e., bounded at infinity with all poles having negative real parts (Desoer and Vidyasagar, 1975).

\section{FRACTIONAL REPRESENTATIONS}

Consider a rational function $G(s)$. By definition, it can be expressed as the ratio

$$
G(s)=\frac{B(s)}{A(s)}
$$

of two qualified rational functions $A$ and $B$.

A well known example is the polynomial description, in which case $A$ and $B$ are coprime polynomials, i.e., polynomials having no roots in common.

Another example is to take for $A$ and $B$ two coprime, proper and stable rational functions. When $G(s)$ is, say,

$$
G(s)=\frac{s+1}{s^{2}+1}
$$


then one can take

$$
A(s)=\frac{s^{2}+1}{(s+\lambda)^{2}}, \quad B(s)=\frac{s+1}{(s+\lambda)^{2}}
$$

where $\lambda$ is a positive real number. We recall that two proper and stable rational functions are coprime if they have no infinite nor unstable zeros in common. Therefore, in the example above, the denominator of $A$ and $B$ can be any strictly Hurwitz polynomial of degree exactly two; if the degree is lower, then $A$ would not be proper and if it is higher, then $A$ and $B$ would have a common zero at infinity. The set of proper and stable rational functions will be denoted by $R_{p s}(s)$. It has the algebraic structure of a ring, in particular a euclidean domain (Vidyasagar, 1985).

\section{FEEDBACK SYSTEMS}

To control a system means altering its dynamics so that a desired behaviour is obtained. This can be done by feedback. A typical feedback system consists of two subsystems, $S_{1}$ and $S_{2}$, connected as shown in Fig. 1

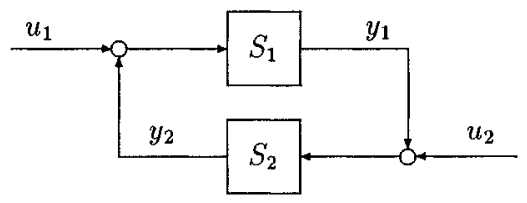

Figure 1 Feedback system

In most applications, it is desirable that the feedback system be BIBO stable in the sense that whenever the inputs $u_{1}$ and $u_{2}$ are bounded in magnitude so too are the outputs $y_{1}$ and $y_{2}$.

In order to study this property, we express the transfer functions of $S_{1}$ and $S_{2}$ as ratios of proper stable rational functions and seek for conditions under which the transfer function of the feedback system is proper and stable.

Thus we write

$$
S_{1}(s)=\frac{B(s)}{A(s)}, \quad S_{2}(s)=-\frac{Y(s)}{X(s)}
$$

where $A, B$ and $X, Y$ are two couples of coprime rational functions from $R_{p s}(s)$. The transfer matrix of the feedback system

$$
\left[\begin{array}{l}
y_{1} \\
y_{2}
\end{array}\right]=\left[\begin{array}{cc}
\frac{S_{1}}{1-S_{1} S_{2}} & \frac{S_{1} S_{2}}{1-S_{1} S_{2}} \\
\frac{S_{1} S_{2}}{1-S_{1} S_{2}} & \frac{S_{2}}{1-S_{1} S_{2}}
\end{array}\right]\left[\begin{array}{l}
u_{1} \\
u_{2}
\end{array}\right]
$$

is then given by

$$
\left[\begin{array}{l}
y_{1} \\
y_{2}
\end{array}\right]=\frac{1}{A X+B Y}\left[\begin{array}{rr}
B X & -B Y \\
-B Y & -A Y
\end{array}\right]\left[\begin{array}{l}
u_{1} \\
u_{2}
\end{array}\right]
$$


We observe that the numerator matrix has all its entries in $R_{p s}(s)$ and that no infinite nor unstable zeros of the denominator can be absorbed in all these entries. We therefore conclude that the four transfer functions belong to $R_{p s}(s)$ if and only if $A X+B Y$ is a unit of $R_{p s}(s)$, i.e., the inverse of $A X+B Y$ belongs to $R_{p s}(s)$.

We illustrate with the example where $S_{1}$ is a differentiator and $S_{2}$ is an invertor such that

We take

$$
S_{1}(s)=s, \quad S_{2}(s)=-1 .
$$

for any real $\lambda>0$ and

$$
A(s)=\frac{1}{s+\lambda}, \quad B(s)=\frac{s}{s+\lambda}
$$

$$
X(s)=1, \quad Y(s)=1 \text {. }
$$

Then

$$
(A X+B Y)^{-1}(s)=\frac{s+\lambda}{s+1}
$$

resides in $R_{p s}(s)$ and hence the closed-loop system is BIBO stable.

To summarize, the fractional representation used should be matched with the goal of the analysis. The analysis is then more transparent and leads to a simple algebraic condition: $A X+B Y$ is a unit of the underlying ring (Desoer et al., 1980).

\section{PARAMETRIZATION OF STABILIZING CONTROLLERS}

The design of feedback control systems consists of the following: given one subsystem, say $S_{1}$, we seek to determine the other subsystem, $S_{2}$, so that the resulting feedback system shown in Fig. 1 meets the design specifications. We call $S_{1}$ the plant and $S_{2}$ the controller.

The primary design specification is some sort of stability. We shall focus on achieving BIBO stability. Any controlller that BIBO stabilizes the plant will be called a stabilizing controller for that plant.

Suppose the plant gives rise to the transfer function

$$
S_{1}(s)=\frac{B(s)}{A(s)}
$$

for some coprime elements $A$ and $B$ of $R_{p s}(s)$. It follows from the foregoing analysis (Kučera, 1979) that a stabilizing controller exists and that all controllers that stabilize the given plant are generated by all solution pairs $X, Y$ with $X \neq 0$ of the Bézout equation

$$
A X+B Y=1
$$

over $R_{p s}(s)$. There is no loss of generality in setting $A X+B Y$ to the identity rather than to an arbitrary unit of $R_{p s}(s):$ this unit is absorbed by $X$ and $Y$ and therefore cancels in forming

$$
S_{2}(s)=-\frac{Y(s)}{X(s)}
$$

The solution set of the Bézout equation can be parametrized (Kučera, 1979) as

$$
X=X^{\prime}+B W, \quad Y=Y^{\prime}-A W
$$


where $X^{\prime}, Y^{\prime}$ is a particular solution of the equation and $W$ is a free parameter, which is an arbitrary function in $R_{p s}(s)$.

The parametrization of the family of all stabilizing controllers for the plant now falls out (Kučera, 1974; Youla et al., 1976) as

$$
S_{2}(s)=-\frac{Y^{\prime}(s)-A(s) W(s)}{X^{\prime}(s)+B(s) W(s)}
$$

where the parameter $W$ varies over $R_{p s}(s)$ while satisfying $X^{\prime}+B W \neq 0$. This condition is not very restrictive, as $X^{\prime}+B W$ can identically vanish for at most one choice of $W$.

As an example, we shall stabilize an integrator plant $S_{1}$. Its transfer function can be expressed as

$$
S_{1}(s)=\frac{\frac{1}{s+1}}{\frac{s}{s+1}}
$$

where $s+1$ is an arbitrarily chosen Hurwitz polynomial of degree one. One stabilizing controller for $S_{1}$ is easy to find, namely

$$
S_{2}(s)=-1 \text {. }
$$

It corresponds to a particular solution $X^{\prime}=1, Y^{\prime}=1$ of the Bézout equation

$$
\frac{s}{s+1} X+\frac{1}{s+1} Y=1 \text {. }
$$

The solution set in $R_{p s}(s)$ of this equation is

$$
X(s)=1+\frac{1}{s+1} W(s), \quad Y(s)=1-\frac{1}{s+1} W(s) .
$$

Hence all controllers $S_{2}$ that BIBO stabilize $S_{1}$ have the transfer function

$$
S_{2}(s)=-\frac{1-\frac{s}{s+1} W(s)}{1+\frac{1}{s+1} W(s)}
$$

where $W$ is any function in $R_{p s}(s)$.

It is clear that the result is independent of the particular fraction taken to represent $S_{1}$. Indeed, if $s+1$ is replaced by another Hurwitz polynomial $s+\lambda$ in the above example, one obtains

which is the same set when

$$
S_{2}(s)=-\frac{\lambda-\frac{s}{s+\lambda} W^{\prime}(s)}{1+\frac{1}{s+\lambda} W^{\prime}(s)}
$$

$$
W^{\prime}(s)=\left(\frac{s+\lambda}{s+1}\right)^{2} W(s)+\frac{s+\lambda}{s+1}(\lambda-1) .
$$

\section{PARAMETRIZATION OF CLOSED-LOOP TRANSFER FUNCTIONS}

The utility of the fractional approach derives not merely from the fact that it provides a parametrization for all controllers that stabilize a given plant in terms of a free parameter 
$W$, but also from the simple manner in which this parameter enters the resulting (proper and stable) closed-loop transfer functions.

In fact,

$$
\left[\begin{array}{l}
y_{1} \\
y_{2}
\end{array}\right]=\left[\begin{array}{cc}
B\left(X^{\prime}+B W\right) & -B\left(Y^{\prime}-A W\right) \\
-B\left(Y^{\prime}-A W\right) & -A\left(Y^{\prime}-A W\right)
\end{array}\right]\left[\begin{array}{l}
u_{1} \\
u_{2}
\end{array}\right]
$$

and we observe that all four transfer functions are linear in the free parameter $W$.

This result serves to parameterize the performance specifications and it is the starting point for the selection of the best controller for the application at hand. The search for $S_{2}$ is thus replaced by a search for $W$. The crucial point is that the resulting selection/optimization problem is linear in $W$ while it is non-linear in $S_{2}$.

\section{OPTIMAL PERFORMANCE}

The performance specifications often involve a norm minimization.

Let us consider the problem of disturbance attenuation. We are given a plant $S_{1}$ having two inputs: the control input $u$ and an unmeasurable disturbance $d$, see Fig. 2.

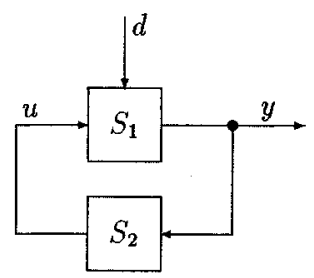

Figure 2 Disturbance attenuation

The objective is to dertermine a BIBO stabilizing controller $S_{2}$ for the plant $S_{1}$ such that the effect of $d$ on the plant output $y$ is minimized in some sense.

We describe the plant by two transfer functions

$$
S_{1 u}(s)=\frac{B(s)}{A(s)}, \quad S_{1 d}(s)=\frac{C(s)}{A(s)}
$$

where $A, B$ and $C$ is a triple of coprime functions from $R_{p s}(s)$. The set of stabilizing controllers for $S_{1}$ is given by the transfer function

$$
S_{2}(s)=-\frac{Y^{\prime}(s)-A^{\prime}(s) W(s)}{X^{\prime}(s)+B^{\prime}(s) W(s)}
$$

where $A^{\prime}, B^{\prime}$ is a coprime fraction over $R_{p s}(s)$ for $S_{1 u}$,

$$
\frac{B(s)}{A(s)}=\frac{B^{\prime}(s)}{A^{\prime}(s)}
$$


and $X^{\prime}, Y^{\prime}$ is a particular solution over $R_{p s}(s)$ of the Bézout equation

$$
A^{\prime} X+B^{\prime} Y=1
$$

such that $X^{\prime}+B^{\prime} W \neq 0$.

The transfer function, $G(s)$, between $d$ and $y$ in a stable feedback system is

$$
G=\frac{S_{1 d}}{1-S_{1 u} S_{2}}=C\left(X^{\prime}+B^{\prime} W\right)
$$

and it is indeed linear in the proper stable rational parameter $W$.

Now suppose that the disturbance $d$ is any function from $L_{\infty}$, i.e., any essentially bounded real function on $R_{+}$. Then (Doyle et al., 1992)

$$
\|y\|_{L_{\infty}} \leq\|G\|_{1}\|d\|_{L_{\infty}}
$$

where

$$
\|G\|_{1}=\int_{0}^{\infty}|g(t)| d t
$$

and $g(t)$ is the impulse response corresponding to $G(s)$. The parameter $W$ can be used to minimize the norm $\|G\|_{1}$ and hence the maximum output amplitude.

If $d$ is stationary white noise, the steady-state output variance equals (Kučera, 1979)

$$
E y^{2}=\|G\|_{2}^{2} E d^{2}
$$

where

$$
\|G\|_{2}^{2}=\int_{0}^{\infty}|g(t)|^{2} d t=\frac{1}{2 \pi j} \oint G(-s) G(s) d s .
$$

The last integral is a contour integral up the imaginary axis and then around an infinite semicircle in the left half-plane. Again, $W$ can be selected so as to minimize the norm $\|G\|_{2}$, thus minimizing the steady-state output variance.

Finally suppose that $d$ is any function from $L_{2}$, i.e., any finite-energy real function on $R_{+}$. Then one obtains (Doyle et al., 1992)

$$
\|y\|_{L_{2}} \leq\|G\|_{\infty}\|d\|_{L_{2}}
$$

where

$$
\|G\|_{\infty}=\sup _{\text {Res }>0}|G(s)| .
$$

Therefore choosing $W$ so as to make the norm $\|G\|_{\infty}$ minimal, one minimizes the maximum output energy.

Here is an illustrative example. The plant is given by

$$
S_{1 u}(s)=\frac{1}{s}, \quad S_{1 d}(s)=1+\frac{1}{s}
$$

and we seek to find a stabilizing controller $S_{2}$ such that $G(s)$ has minimum $\infty$-norm.

We write

$$
A(s)=\frac{s}{s+1}, \quad B(s)=\frac{1}{s+1}, \quad C(s)=1
$$

and recall that the set of stabilizing controllers is given by

$$
S_{2}(s)=-\frac{1-\frac{s}{s+1} W(s)}{1+\frac{1}{s+1} W(s)}
$$


where $W$ is a free parameter in $R_{p s}(s)$. Then

$$
G(s)=\frac{S_{1 d}(s)}{1-S_{1 u}(s) S_{2}(s)}=1+\frac{1}{s+1} W(s)
$$

so that the least norm $\|G\|_{\infty}=0$ is achieved by $W(s)=-(s+1)$. This parameter, however, does not belong to $R_{p s}(s)$. So we approximate it by

$$
W_{\varepsilon}(s)=-\frac{s+1}{\varepsilon s+1}
$$

for any real $\varepsilon>0$. Then

$$
G_{\varepsilon}(s)=\frac{\varepsilon s}{\varepsilon s+1}
$$

and the least $\infty$-norm attainable by a proper and stable rational parameter $W_{\varepsilon}$ is $\left\|G_{\varepsilon}\right\|_{\infty}=1$. The optimal stabilizing controller guarantees $\|y\|_{L_{2}} \leq\|d\|_{L_{2}}$ for any $d \in L_{2}$.

\section{ROBUST STABILIZATION}

The actual plant can differ from its nominal model. We suppose that a nominal plant description is available together with a description of the plant uncertainty. The objective is to design a controller that stabilizes the nominal plant as well as all plants lying within the specified domain of uncertainty. Such a controlller is said to robustly stabilize the plant.

The plant uncertainty can be modelled conveniently in terms of the fractional representation over $R_{p s}(s)$. We endow $R_{p s}(s)$ with the $\infty$-norm: for any function $F(s)$ from $R_{p s}(s)$

$$
\|F\|_{\infty}=\sup _{\text {Re } s>0}|F(s)| .
$$

For any two such function, $F_{1}(s)$ and $F_{2}(s)$, we define

$$
\left\|\left[\begin{array}{ll}
F_{1} & F_{2}
\end{array}\right]\right\|_{\infty}=\left\|\left[\begin{array}{l}
F_{1} \\
F_{2}
\end{array}\right]\right\|_{\infty}=\sup _{\operatorname{Re} s>0}\left(\left|F_{1}(s)\right|^{2}+\left|F_{2}(s)\right|^{2}\right)^{1 / 2}
$$

Let $S_{10}$ be a nominal plant giving rise to a transfer function

$$
S_{10}(s)=\frac{B(s)}{A(s)}
$$

where $A$ and $B$ are coprime functions from $R_{p s}(s)$. We denote $S_{1}(A, B, \mu)$ the family of plants having transfer functions

$$
S_{1}(s)=\frac{B(s)+\Delta B(s)}{A(s)+\Delta A(s)}
$$

where $\Delta A$ and $\Delta B$ are functions from $R_{p s}(s)$ such that

$$
\left\|\left[\begin{array}{ll}
\Delta A & \Delta B
\end{array}\right]\right\|_{\infty}<\mu
$$

for some non-negative real number $\mu$.

Now, let $S_{2}$ be a BIBO stabilizing controller for $S_{10}$. Therefore

$$
S_{2}(s)=-\frac{Y^{\prime}(s)-A(s) W(s)}{X^{\prime}(s)+B(s) W(s)}
$$


where $A X^{\prime}+B Y^{\prime}=1$ and $W$ is an element of $R_{p s}(s)$. Then $S_{2}$ will BIBO stabilize all plants from $S_{1}(A, B, \mu)$ if and only if

$$
(A+\Delta A)\left(X^{\prime}+B W\right)+(B+\Delta B)\left(Y^{\prime}-A W\right)=1+\left[\begin{array}{ll}
\Delta A & \Delta B
\end{array}\right]\left[\begin{array}{c}
X^{\prime}+B W \\
Y^{\prime}-A W
\end{array}\right]
$$

is a unit of $R_{p s}(s)$. This is the case whenever

$$
\left\|\left[\begin{array}{ll}
\Delta A & \Delta B
\end{array}\right]\left[\begin{array}{l}
X^{\prime}+B W \\
Y^{\prime}-A W
\end{array}\right]\right\|_{\infty}<1
$$

so we have the following condition of robust stability (Vidyasagar, 1985):

$$
\mu\left\|\left[\begin{array}{c}
X^{\prime}+B W \\
Y^{\prime}-A W
\end{array}\right]\right\|_{\infty} \leq 1
$$

The best controller that robustly stabilizes the plant corresponds to the parameter $W$ that minimizes the $\infty$-norm above.

To illustrate, consider the family of plants

$$
S_{1}=\left(\frac{s}{s+1}, \frac{1}{s+1}, \mu\right)
$$

around the nominal model

$$
S_{10}(s)=\frac{1}{s}
$$

This family accommodates, among other things, gain perturbations and stable secondorder dynamics of the form

$$
S_{1}(s)=\frac{1+\mu}{s(1+\varepsilon s)} .
$$

All controllers that BIBO stabilize the nominal plant $S_{10}$ are given by

$$
S_{2}(s)=-\frac{1-\frac{s}{s+1} W(s)}{1+\frac{1}{s+1} W(s)}
$$

where $W$ is a free parameter in $R_{p s}(s)$.

Suppose we wish to obtain a robust proportional controller, $S_{2}(s)=-\lambda$. Then

$$
W(s)=(1-\lambda) \frac{s+1}{s+\lambda}
$$

and $\lambda>0$. The norm

$$
\left\|\left[\begin{array}{l}
1+\frac{1}{s+1} W \\
1-\frac{s}{s+1} W
\end{array}\right]\right\|_{\infty}=\sqrt{1+\lambda^{2}}\left\|\frac{s+1}{s+\lambda}\right\|_{\infty}
$$

is calculated to be

$$
N(\lambda)=\left\{\begin{array}{lll}
\frac{\sqrt{1+\lambda^{2}}}{\lambda}, & \text { if } & 0<\lambda<1 \\
\sqrt{1+\lambda^{2}}, & \text { if } & \lambda \geq 1 .
\end{array}\right.
$$

Thus our proportional controller is robust if and only if

$$
N(\lambda) \leq \frac{1}{\mu} .
$$


Which proportional controller will maximize the stability margin $\mu$ ? The one which minimizes $N(\lambda)$. Since

$$
\min _{\lambda>0} N(\lambda)=\sqrt{2}
$$

is attained by $\lambda=1$, which corresponds to $W(s)=0$, the consequent controller $S_{2}(s)=-1$ achieves the stability margin as large as

$$
\mu=\frac{1}{\sqrt{2}}
$$

\section{REFERENCES}

Desoer, C. A. and M. Vidyasagar (1975) Feedback Systems: Input-Output Properties. Academic Press, New York.

Desoer, C. A., R. W. Liu, J. Murray and R. Saeks (1980) Feedback systems design: The fractional representation approach to analysis and synthesis. IEEE Trans. Aut. Control, AC-25, 399-412.

Doyle, J. C., B. A. Francis and A. R. Tannenbaum (1992) Feedback Control Theory. Macmillan, New York.

Kučera, V. (1974) Closed-loop stability of discrete linear single-variable systems. Kybernetika, 10, 146-171.

Kučera, V. (1979) Discrete Linear Control: The Polynomial Equation Approach. Wiley, Chichester.

Kučera, V. (1993) Diophantine equations in control - A survey. Automatica, 29, 13611375.

Vidyasagar, M. (1985) Control System Synthesis: A Factorization Approach. MIT Press, Cambridge, MA.

Youla, D. C., J. J. Bongiorno and H. A. Jabr (1976) Modern Wiener-Hopf design of optimal controllers, Part I: The single-input case. IEEE Trans. Aut. Control, AC-21, 3-14. 